\title{
Fuzzy Reliability Based Bow Tie Analysis of Coal Mine Water Inrush Risks
}

\author{
Cong Zhang, Huaying Wang* and Yixin Wei \\ Department of Mineral Resources Engineering, University of Science and Technology Beijing, Beijing, China
}

\begin{abstract}
Water inrush is a serious geological accident in underground coal mines. This paper focuses on the method of the bow tie analysis based on fuzzy reliability to assess the risk of water inrush in coal mines. First, post failure probability and repair probability of initiating events are divided into 5 levels. Then triangular fuzzy numbers are adopted to quantify experts' language and to acquire the reliability rate which close to the actual values by defuzzification and calculation. According to dynamic bow-tie analysis model, using the method of moments to fit the reliability time of events by Weibull distribution and offering the random sampling rule of failure and repair time for illustrating the feasibility of fuzzy reliability evaluation. To demonstrate the effectiveness of the proposed assessment approach, bow tie analysis model of water inrush in coal mines is taken as an example.
\end{abstract}

Keywords: water inrush in coal mines, bow tie analysis, fuzzy reliability, Weibull distribution

\section{Introduction}

Dynamic bow tie (DBT) analysis is composed of dynamic fault tree and dynamic event tree analysis, and considers the impact of the time factor upon risk assessment of a system. There are many algorithms applied to calculation of the probabilities of node accident and accident consequence of dynamic bow tie analysis: Hidden Markov Model, Bayesian Network (Khakzad et al 2013), Integral Algorithm (Amari et al 2003) and Monte Carlo Simulation (Kim et al 2016). In terms of simulated time and practicability, the Monte Carlo Simulation has an advantage over other methods. DBT requires mass data in quantitative analysis, including the failure rates and the repair rates of the basic events of the Fault Tree (FT) and the subsequent events of the Event Tree (ET) at different time points, i.e., the distribution of failure time and repair time. In practical analysis, it is hard to obtain a large amount of exact historical statistical data, which is a major problem of risk assessment.

Due to the difficulty of obtaining the exact data and historical failure data of the basic events and the subsequent events, the FT and ET analysis is stuck with the study of minimal cut sets, minimal diameter sets, structural importance and qualitative analysis (Liu and Zhang 2010, Yan et al 2012, Wang et al 2009, Li and Wang 2011). This paper will use fuzzy numbers and the defuzzification method to transform the experts' language into the failure rates and repair rates of basic events and subsequent events, solving the problem that it is difficult to obtain a large amount of historical statistical data, and will use the method of moment to conduct Weibull fitting of the reliability time to provide the random sampling criteria of event failure and repair time in Monte Carlo simulation.

\section{Fuzzy Reliability Calculation}

System reliability is defined as the ability of the product to perform its intended function under the specified conditions and time. This ability is assessed by the failure or repair situation. For example, failure time and repair time are introduced to reliability evaluation of a power grid (Zhao et al 2013). This paper uses failure rate, failure time, repair rate and repair time to describe the reliability, and uses triangular fuzzy numbers to represent probability values of occurrence of the events which do not allow the acquisition of the exact historical data.

\subsection{Establishment of reliability evaluation level}

To assess the basic and subsequent events of risks, experienced experts are needed. However, it is hard for even the experts to give accurate mathematical representation for failure events and repair rate. The language of the experts' subjective description is fuzzy, like "very low" and "higher". The actual reliability probabilities reflected by the accident fuzzy reliability evaluation languages of different industries are not the same. For instance, the failure rate of a coal mine water inrush accident and related events is lower than accidents (like electrical appliance accidents) of other industries. The probability range is different in different types of accidents. In the nuclear industry, "very low" can represent the failure rate which is smaller than $10^{-8}$.

The triangular fuzzy numbers are represented by three parameters, and the membership function thereof is: The triangular fuzzy numbers is represented by three parameters $\underline{A}=(\mathrm{a}, \mathrm{c}, \mathrm{b})$, and membership function is:

$$
\mu_{\underline{A}}(\mathrm{x})=\left\{\begin{array}{cc}
(x-a) /(c-a) & (a \leq x \leq c) \\
(b-x) /(b-c) & (\mathrm{c} \leq \mathrm{x} \leq \mathrm{b}) \\
0 & \text { otherwise }
\end{array}\right.
$$

\footnotetext{
* Corresponding Author: H.Y. Wang, 252110737@163.com, phone: +86 10-62334756
} 
where $\underline{A}$ is the fuzzy set in the domain, $\mu_{\underline{A}}(\mathrm{x})$ is the membership function of $\mathrm{x}$ to the fuzzy set $\underline{A}$.

In order to facilitate expert evaluation, the fuzzy language is divided into 5 levels: "Very Low", "Low", "Medium", "High" and "Very High". Each level has no clear boundaries. The failure rate and the repair rate of events are less likely to be "Very High" and "Very Low", followed by "High" and "Low", and are most likely to be "Medium". First of all, the triangular fuzzy numbers of "Medium" are determined as $(0.3,0.5,0.7)$. Then, based on symmetry and the principle that the interval width decreases in sequence, other levels of fuzzy numbers are determined. The finalized triangular fuzzy numbers are as follows:

$$
\begin{aligned}
& \mu_{\text {very high }}(x)=\mu_{1}(x)=(0.00,0.05,0.10) \\
& \mu_{\text {low }}(x)=\mu_{2}(x)=(0.08,0.23,0.38) \\
& \mu_{\text {medium }}(x)=\mu_{3}(x)=(0.30,0.50,0.70) \\
& \mu_{\text {high }}(x)=\mu_{4}(x)=(0.62,0.77,0.92) \\
& \mu_{\text {very high }}(x)=\mu_{5}(x)=(0.90,0.95,1.00)
\end{aligned}
$$

Suppose $\mathrm{m}$ events need to be assessed by $\mathrm{n}$ experts in a bow tie analysis model, the $\mathrm{m}^{*} \mathrm{n}$ matrix is as follows:

$$
A=\left[\begin{array}{cccc}
\mu_{1}{ }^{1} & \mu_{1}{ }^{2} & \cdots & \mu_{1}^{n} \\
\mu_{2}{ }^{1} & \mu_{2}{ }^{2} & \cdots & \mu_{2}{ }^{n} \\
\vdots & \vdots & \ddots & \vdots \\
\mu_{m}{ }^{1} & \mu_{m}{ }^{2} & \cdots & \mu_{m}{ }^{n}
\end{array}\right]
$$

Where $\mu_{1}^{1}$ is the triangular fuzzy numbers corresponding to the evaluative language of a first event by a first expert. For example, if the evaluative language is "low", $\mu_{1}^{1}=\mu_{2}(x)=(0.08,0.23,0.38)$.

\subsection{Calculation of fuzzy reliability probability}

According to the experts' fuzzy evaluative language and the corresponding triangular fuzzy numbers, defuzzification is used to calculate the score that best represents the fuzzy language. The fuzzy score is transformed into the reliability probability that is close to the true value, using the empirical formula.

\subsubsection{Defuzzification fuzzy evaluation language}

There are a number of defuzzification methods for triangular fuzzy numbers, including: max-average, center of area and so on. This paper adopts the centroid point defuzzification method, wherein the fuzzy evaluation value is $r=\frac{1}{3}(a+b+c)$. Centroid point defuzzification is conducted on (7) to lead to the score $\mathrm{R}$ of $\mathrm{m}$ events by $\mathrm{n}$ experts.

$$
R=\left[\begin{array}{cccc}
r_{1}{ }^{1} & r_{1}{ }^{2} & \cdots & r_{1}{ }^{n} \\
r_{2}{ }^{1} & r_{2}{ }^{2} & \cdots & r_{2}{ }^{n} \\
\vdots & \vdots & \ddots & \vdots \\
r_{m}{ }^{1} & r_{m}{ }^{2} & \cdots & r_{m}{ }^{n}
\end{array}\right]=\left[\begin{array}{cccc}
r\left(\mu_{1}{ }^{1}\right) & r\left(\mu_{1}{ }^{2}\right) & \cdots & r\left(\mu_{1}{ }^{n}\right) \\
r\left(\mu_{2}{ }^{1}\right) & r\left(\mu_{2}{ }^{2}\right) & \cdots & r\left(\mu_{2}{ }^{n}\right) \\
\vdots & \vdots & \ddots & \vdots \\
r\left(\mu_{m}{ }^{1}\right) & r\left(\mu_{m}{ }^{2}\right) & \cdots & r\left(\mu_{m}{ }^{n}\right)
\end{array}\right]
$$

Where $r_{1}^{1}$ denotes the fuzzy evaluation value of a first event by a first expert, and $r\left(\mu_{1}^{1}\right)$ is the defuzzification function of the fuzzy evaluation value of the first event by the first expert.

\subsubsection{Calculation of actual probability of reliability}

The fuzzy value $(0 \sim 1)$ only denotes the comparison value in the range of event failure rate or repair rate, and is not the real reliability probability value.

$$
p=\left\{\begin{array}{cc}
\frac{1}{10^{y}} & r \neq 0 \\
0 & r=0
\end{array}\right.
$$

where

$$
\mathrm{y}=\left(\frac{1-r}{r}\right)^{1 / 3} \times 2.301
$$

and $\mathrm{p}$ is the actual probability value of the event reliability, $\mathrm{r}$ is the fuzzy evaluation value. From Eq. (1-9) we find the actual reliability probability close to the real value.

The fuzzy evaluation value is used to transform Eq. (19) into the actual event failure rate and repair rate, and the matrix obtained is as follows:

$$
\mathrm{P}=\left[\begin{array}{cccc}
p_{1}{ }^{1} & p_{1}{ }^{2} & \cdots & p_{1}{ }^{n} \\
p_{2}{ }^{1} & p_{2}{ }^{2} & \cdots & p_{2}{ }^{n} \\
\vdots & \vdots & \ddots & \vdots \\
p_{m}{ }^{1} & p_{m}{ }^{2} & \cdots & p_{m}{ }^{n}
\end{array}\right]=\left[\begin{array}{cccc}
p\left(\mathrm{r}_{1}{ }^{1}\right) & p\left(\mathrm{r}_{1}{ }^{2}\right) & \cdots & p\left(\mathrm{r}_{1}{ }^{n}\right) \\
p\left(\mathrm{r}_{2}{ }^{1}\right) & p\left(\mathrm{r}_{2}{ }^{2}\right) & \cdots & p\left(\mathrm{r}_{2}{ }^{n}\right) \\
\vdots & \vdots & \ddots & \vdots \\
p\left(\mathrm{r}_{m}{ }^{1}\right) & p\left(\mathrm{r}_{m}{ }^{2}\right) & \cdots & p\left(\mathrm{r}_{m}{ }^{n}\right)
\end{array}\right]
$$

where $p_{1}^{1}$ is the actual reliability probability of a first event evaluated by a first expert; ${ }^{p\left(\mathrm{r}_{1}^{1}\right)}$ is a function to obtain the actual reliability probability from the fuzzy evaluation value of the first event value by the first expert.

\section{Reliability Time Distribution}

The traditional bow tie analysis just needs the reliability probability value of an event. Through the FT and ET analysis, the probabilities of node accident and accident consequence are obtained. However, the dynamic bow tie analysis not only includes Boolean logic relation but also includes dynamic logic gates, and some events' reliability changes over time. In order to simulate the dynamic bow tie analysis model, we need to obtain the reliability time distribution of the reliability variable events, i.e., failure time and repair time distribution.

\subsection{Calculating reliability time base on reliability probability}

An event's failure rate $f p$ at time t means: the probability $f p$ of occurrence of the accident described by the event in a unit time after a time $t$ when the system has operated till the time $t$ (Xu et al 2012). In other words, the accident described by the event occurs $f p$ times in one unit time. Usually, $f p<=1$, which means that the accident described by the event occurs once in $1 / f$ unit time. In the same way, the accident repair described by the event occurs once in $1 / r p$ ( $r p$ is the repair rate) unit time. According to above reasoning, the system reliability time is calculated as follows: 


$$
T=\left[\begin{array}{cccc}
t_{1}{ }^{1} & t_{1}{ }^{2} & \cdots & t_{1}{ }^{n} \\
t_{2}{ }^{2} & t_{2}{ }^{2} & \cdots & t_{2}{ }^{n} \\
\vdots & \vdots & \ddots & \vdots \\
t_{m}{ }^{1} & t_{m}{ }^{2} & \cdots & t_{m}{ }^{n}
\end{array}\right]=\left[\begin{array}{cccc}
\frac{1}{p_{1}{ }^{1}} & \frac{1}{p_{1}{ }^{2}} & \cdots & \frac{1}{p_{1}{ }^{n}} \\
\frac{1}{p_{2}{ }^{1}} & \frac{1}{p_{2}{ }^{2}} & \cdots & \frac{1}{p_{2}{ }^{n}} \\
\vdots & \vdots & \ddots & \vdots \\
\frac{1}{p_{m}{ }^{1}} & \frac{1}{p_{m}{ }^{2}} & \cdots & \frac{1}{p_{m}{ }^{n}}
\end{array}\right]
$$

where $t_{1}^{1}$ denotes the reliability time of a first event evaluated by a first expert.

\subsection{Weibull distribution fitting of reliability time}

The hypothetic distribution of reliability analysis includes: exponential distribution, Gamma distribution and Weibull distribution. The exponential distribution is most commonly used. However, the exponential distribution has memory (when it is known that the basic event keeps the original state for s hours, $f(t)=f(s+t))$, wherein the failure rate is a constant and the application thereof is limited). In recent years, research shows that the rule of product failure complies with the Weibull distribution (Behnia et al 2016). A bathtub curve comprises three parts: early failures, random failures and wear-out failures, which correspond to shape parameters $\beta<1, \beta=1$ and $\beta>1$ of Weibull, respectively. The unsafe factors can be divided into "Human", "Machine", "Environment" and "Management". The "Human" and "Machine" hazards resemble the rule of "Machine" wherein the failure rate decreases in the early stage, remains substantially constant during the middle stage, and finally increases. Therefore, the failure and repair time of events is in accordance with Weibull distribution fitting in this paper.

The probability density formula of 2-parameters Weibull distribution is:

$$
f(x)=\frac{\beta}{\eta}\left(\frac{x}{\eta}\right)^{\beta-1} e^{-\left(\frac{x}{\eta}\right)^{\beta}}
$$

the distribution function is written as:

$$
\mathrm{F}(x)=1-e^{-\left(\frac{x}{\eta}\right)^{\beta}}
$$

$\eta$ is the Weibull distribution scale parameter, $\beta$ is Weibull distribution shape parameter.

There are many parameter estimation methods for 2parameters Weibull distribution, such as graphic method, maximum likelihood estimation, moment method, least square method and empirical approach (Cheng and Sheu 2016, Saleh et al 2012). Therefore, this paper uses the moment method (MoM) to get the event reliability time based on parameter estimation of Weibull distribution. The MoM estimates Weibull distribution parameters in the following way:

$$
\begin{aligned}
& \beta=\left(\frac{\sigma}{\bar{t}}\right)^{-1.086} \\
& \eta=\frac{\bar{t}}{\Gamma(1+1 / \beta)}
\end{aligned}
$$

Where:

$$
\bar{t}=\frac{1}{n} \sum_{i=1}^{n} t_{i}
$$

$$
\sigma=\sqrt{\frac{1}{n-1} \sum_{i=1}^{n}\left(\mathrm{t}_{i}-\bar{t}\right)^{2}}
$$

Weibull parameter estimate Matrix T in Eq. (2-1) to get the distribution parameter of failure and repair time. Shape parameter determinant is $B=\left(\beta_{1}, \cdots, \beta_{m}\right)^{T}$, scale parameter determinant is $N=\left(\eta_{1}, \cdots, \eta_{m}\right)^{T}$.

\section{Fuzzy Reliability Evaluation}

The number of deaths in water inrush accidents is second in all coal mine accidents, and the number of occurrences is third. Therefore, risk assessment of water inrush is very important. However, quantitative analysis needs mass data, but it is impossible to obtain all the accurate data in reality or theoretically.

\subsection{Building the dynamic bow tie model}

The bow tie analysis model is built with the water inrush point in mine as the node of bow tie analysis, using FT to identify hazards of water inrush risks and ET to show the evolution of water inrush accident. Base on the occurrence mechanism of water inrush accident in mine, water inrush accident in coal mine is a state that draining ability of a mine cannot control the mine water in a reasonable range when abnormal water increases, and the emergency measures fail. According to the characteristics of water inrush in coal mine and survey of the literature, the 3-level FT model is built (Gao 2010). In the 3-level FT model, "sufficient aggregation of the source of water inrush" is the prerequisite of water inrush occurrence. In China, the sources of water inrush are mainly surface water, pore water, fracture water, karst and goaf water (Wu 2013, State Administration of Work Safety 2011a, State Administration of Work Safety 2011b). Expert advice, accident investigation and literature review are adopted to analyze a water inrush point accident by ET. There are usually five key events after accident occurrence, including: breaking through the inrush zone, flooding the horizontal tunnel, flooding the local lowest point, flooding the shaft station and flooding the shaft or inclined shaft. The local lowest point means that this point has no other adjacent points except the inflow point or the elevations of the other adjacent points are higher than that of this point (Li 2012).

Dynamic analysis is conducted on the FT and ET model, and a dynamic bow tie analysis model is built. The model includes one SEQ and one PAND. In Figure 1, there are 22 hazards of water inrush in coal mine. Nine factors (X6、X7、 X8、X12、X13、X15、Xc17、X19、X21) are human factors, two (X18、X20) are machine factors, seven (X1、 $\mathrm{X} 2 、 \mathrm{X} 3 、 \mathrm{X} 4 、 \mathrm{X} 5 、 \mathrm{X} 9 、 \mathrm{X} 10)$ are environment factors and four (X11、X14、X19、X22) are management factors. Once the water inrush happens, there will be six different consequences $(\mathrm{C} 1 、 \mathrm{C} 2 、 \mathrm{C} 3 、 \mathrm{C} 4 、 \mathrm{C} 5 、 \mathrm{C} 6)$.

\subsection{Reliability time Weibull distribution}

The hazards of environment factors $\mathrm{X} 1 、 \mathrm{X} 2 、 \mathrm{X} 3 、 \mathrm{X} 4$ 、 X5、X9、X10 can be obtained from statistical data, but the 


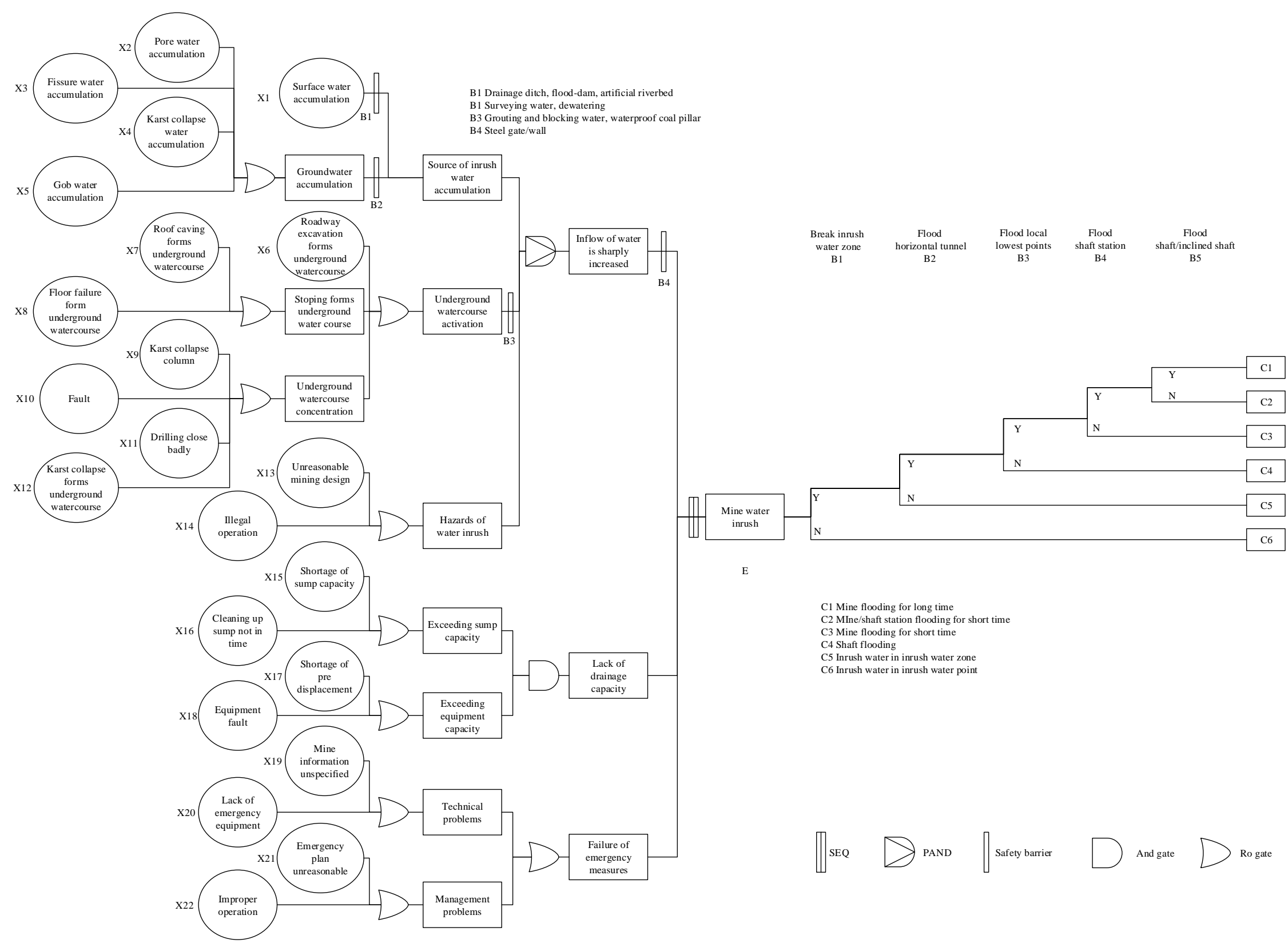

Figure 1. The bow-tie graph of mine water inrush. 
data of other hazards and subsequent events cannot be obtained or accurate data thereof is hard to obtain. According the logical relationship between SEQ and PAND, with the treatment process in Part 1 and Part 2, fuzzy reliability evaluation is conducted to obtain parameter values of failure time and repair time Weibull distribution.

According to the fuzzy reliability evaluation approach, ten experts of water prevention and control in coal mine are first invited to assess the failure rate and repair rate of basic events and subsequent events of a water inrush part at a coal mine water inrush point.

According to formulas (2) - (6), the experts' assessment is transformed into matrix $\mathrm{A}$, to obtain the corresponding triangular fuzzy numbers. Next, experts' triangular fuzzy evaluation numbers are defuzzified. A number of defuzzification methods are available, including centroid method, center of gravity, mean max membership and so on. Through the pilot calculation, it is found that center of gravity (COG) is the best for water inrush risk assessment in coal mine. Through defuzzification of matrix A, experts' scores can be obtained, i.e., matrix R (8). In order to make the fuzzy rate close to the real failure rate and repair rate, (10) is used to obtain the real failure rate and repair rate matrix $\mathrm{P}$ of basic events and subsequent events, like (11). In dynamic bow tie analysis, the failure time and repair time of basic events and subsequent events need to be obtained, and the bow tie model is simulated by Monte Carlo. Hence, we need failure time and repair time distribution of basic events and subsequent events to provide the failure time and repair time random sampling principle for Monte Carlo simulation.

In order to conduct Weibull distribution fitting of failure time and repair time, the matrix method is used to estimate the shape parameter and the scale parameter. Formulas (15) and (18) are used to estimate the failure time and the repair time of basic events, respectively. We can obtain shape parameter determinant B and scale parameter determinant $\mathrm{N}$.

In the dynamic bow tie graph of water inrush in coal mine, SEQ appears in the first level of FT on the left of the node. Thus, failure time distribution needs to be considered in all the basic events of water inrush in mine. The logical relationship of subsequent events is similar to PAND in the part of FT on the right of the node, so the failure time distribution also needs to be considered. The failure rates of $\mathrm{X} 1, \mathrm{X} 2, \mathrm{X} 3, \mathrm{X} 4, \mathrm{X} 5, \mathrm{X} 9$ and X10 are constants and can be obtained through statistical data. The above events, once happening, cannot be repaired, and the repair rate is zero. According the logical relationship between SEQ and PAND, one only needs to get the repair time distribution of X15, X16, X17 and X18 to conduct Monte Carlo simulation on a bow tie analysis model of water inrush in coal mine. Based on above analysis, Weibull distribution parameters of failure time and repair time of some basic events and subsequent events in bow tie analysis of water inrush in coal mine are obtained, as shown in Table 1.

In Table 1, scale parameters of failure time and repair time Weibull distribution are greatly different. For example, the scale parameter of X11 "poor drilling sealed off" is
19.7467, which means most of the failure time is in the 19.7467 unit time. The scale parameter of X12 "karst collapse by mine drainage" is 6755.016 , which means most of the failure time is in the 6755.016 unit time. The above results demonstrate that the failure rates and repair rates of different events at different time points are very different. Past static bow tie analysis is on the premise that the event reliability is constant, which is not in conformity with the actual situation. Dynamic bow tie analysis considers the dynamic effect of the time factor on the system, which is more accordant with the actual situation.

Table 1. Weibull distribution parameter.

\begin{tabular}{cccccc}
\hline Basic/subsequent & \multicolumn{2}{c}{ Failure time } & \multicolumn{2}{c}{ Repair time } \\
events & $\beta$ & $\eta$ & $\beta$ & $\eta$ \\
\hline $\mathrm{X}_{6}$ & 1.5037 & 23.1632 & - & - \\
\hline $\mathrm{X}_{7}$ & 1.3792 & 128.3423 & - & - \\
\hline $\mathrm{X}_{8}$ & 1.3437 & 1896.993 & - & - \\
\hline $\mathrm{X}_{11}$ & 1.3211 & 19.7467 & - & - \\
\hline $\mathrm{X}_{12}$ & 5.3633 & 6755.016 & - & - \\
\hline $\mathrm{X}_{13}$ & 1.1812 & 877992.3 & - & - \\
\hline $\mathrm{X}_{14}$ & 1.5037 & 23.1632 & - & - \\
\hline $\mathrm{X}_{15}$ & 1.1812 & 87992.3 & 1.0479 & 1478.376 \\
\hline $\mathrm{X}_{16}$ & 1.6275 & 149.4624 & 3.7119 & 84.388 \\
\hline $\mathrm{X}_{17}$ & 1.1812 & 87992.3 & 5.8611 & 1113.253 \\
\hline $\mathrm{X}_{18}$ & 5.3633 & 1755.016 & 2.6191 & 57.782 \\
\hline $\mathrm{X}_{19}$ & 1.1854 & 106.9579 & - & - \\
\hline $\mathrm{X}_{20}$ & 5.3633 & 6755.01 & - & - \\
\hline $\mathrm{X}_{21}$ & 1.1812 & 877992.3 & - & - \\
\hline $\mathrm{X}_{22}$ & 1.1051 & 1540.45 & - & - \\
\hline $\mathrm{B}_{1}$ & 1.7562 & 26.5358 & - & - \\
\hline $\mathrm{B}_{2}$ & 5.3633 & 6755.01 & - & - \\
\hline $\mathrm{B}_{3}$ & 1.3793 & 128.3423 & - & - \\
\hline $\mathrm{B}_{4}$ & 1.1051 & 1540.46 & - & - \\
\hline $\mathrm{B}_{5}$ & 1.5323 & 1170221.0 & - & - \\
\hline & & & &
\end{tabular}

\section{Conclusions}

It is hard to obtain the accurate historical data in actual risk management. The bow tie analysis of water inrush in coal mine needs the qualitative description from experts, for example. Fuzzy reliability evaluation calculates the reliability data of dynamic bow tie analysis based on basic event and subsequent event reliability probabilities in a dynamic bow tie analysis model characterized by triangular fuzzy numbers, and failure time and repair time of basic events and subsequent events fitted by Weibull distribution. To verify the feasibility of fuzzy reliability evaluation, the bow tie analysis of water inrush in coal mine is taken as an example. The result shows that the scale parameters of failure time and repair time Weibull distribution of basic events and subsequent events differ greatly. The case study shows that dynamic bow tie analysis is more practical than traditional bow tie analysis, and that fuzzy reliability evaluation is an effective method for calculating the event reliability value when the historical data is hard to obtain. 


\section{References}

Amari, S., G. Dill and E. Howald, 2003. A new approach to solve dynamic fault trees. Reliability and Maintainability Symposium. Annual. IEEE, 374 - 379. DOI: 10.1109/RAMS.2003.1182018.

Behnia, A., N. Ranjbar, H. K. Chai, \& M. Masaeli, 2016. Failure prediction and reliability analysis of ferrocement composite structures by incorporating machine learning into acoustic emission monitoring technique. Construction and Building Materials, 122: 823 - 832. DOI: 10.1016/j.conbuildmat.2016.06.130

Cheng, Y. and S. Sheu, 2016. Robust estimation for weibull distribution in partially accelerated life tests with early failures. Quality and Reliability Engineering International. 32: 2207 - 2216. DOI: 10.1002/qre.1928

Gao, Y., 2010. Application of fault tree analysis to establish safety checklist for groundwater inrush in underground coalmines. Coal Geology \& Exploration. 06: 57 - 61.

Khakzad, N., F. Khan and P. Amyotte, 2013. Dynamic safety analysis of process systems by mapping bow-tie into bayesian network. Process Safety and Environmental Protection, 91(1): 46 - 53. DOI: 10.1016/j.psep.2012.01.005

Kim, J., Y. Noh, K. Chang and D. Chang, 2016. Determination of hydrate inhibitor injection rate for flowlines based on Monte Carlo method. Journal of Loss Prevention in the Process Industries, 44: 62 - 72. DOI: $10.1016 /$ j.jlp.2016.08.012

Li, K. and Z. Wang, 2011. The application of fault tree of gas in mine. Energy Technology and Management. 03: 110 - 111. DOI: 10.3969/j.issn.1672-9943.2011.03.045

Li, X., 2012. Dynamic visualization of water inrushes and emergency responses in underground mines. Ph.D. Thesis, University of Science and Technology, Beijing.
Liu, G. and L. Zhang, 2010. The application of fault tree of tunnel engineering in geological survey during flood accident about lead-zinc deposit in huajiaoshuping, shenlongjia forestry region. Resources Environment \& Engineering, 02: $200 \quad-\quad 202 . \quad$ DOI: 10.16536/j.cnki.issn.1671-1211.2010.02.013.

Saleh, H., E.A. Aly and S. Abdel-Hady, 2012. Assessment of different methods used to estimate weibull distribution parameters for wind speed in zafarana wind farm, Suez Gulf, Egypt. Energy, 44(1): 710 - 719. DOI: 10.1016/j.energy.2012.05.021.

State Administration of Work Safety, 2011a. Provisions on prevention and control of water in coal mines. China Coal Industry Publishing, Beijing.

State Administration of Work Safety, 2011b. Safety regulations in coal mine. China Coal Industry Publishing, Beijing.

Wang, C., Y. Sun and Y. Hang, 2009. Application of fault tree analysis to risk assessment of potential waterinrush hazards in coal mining. Chinese Journal of Rock Mechanics and Engineering. 02: 398 - 305.

$\mathrm{Wu}, \mathrm{Q}$., 2013. Prevention and control of coal mine water. China Coal Industry Publishing, Beijing, 41 - 44.

Xu, J., D. Sun, Y. Fan and Q. Ren, 2012. Accident risk probability for "three highs" gas fields based on fault tree analysis. Systems Engineering - Theory \& Practice, 4: 877 - 884.

Yan, C., J. Ji, W. Liu and S. Chen, 2012. Fault tree analysis of water inrush in coal mine. Modern Mining, 11: 141 143.

Zhao, Y., Y. Guo and K. Xie, 2013. Research on probability distribution characteristics of bulk power system reliability considering parameter uncertainty. Power System Technology, 08: 2165 - 2172. DOI: 10.13335/j.1000-3673.pst.2013.08.018 